\title{
Correlation between cortisol level and serotonin uptake in patients with chronic stress and depression
}

\author{
GUSTAVO E. TAFET \\ Weizmann Institute of Science, Rehovot, Israel, \\ and Instituto de Investigaciones Bioquímicas, Fundación Campomar, \\ Universidad de Buenos Aires, Argentina
}

\author{
VICTOR P. IDOYAGA-VARGAS, DENISE P. ABULAFIA, and JORGELINA M. CALANDRIA \\ and Instituto de Investigaciones Bioquímicas, Fundación Campomar, \\ Universidad de Buenos Aires, Buenos Aires, Argentina
}

SILVIA S. ROFFMAN and AMABELLA CHIOVETTA

Hospital Zubizarreta, Buenos Aires, Argentina

and

MEIR SHINITZKY

Weizmann Institute of Science, Rehovot, Israel

\begin{abstract}
In a recent study (Tafet, Toister-Achituv, \& Shinitzky, 2001), we demonstrated that cortisol induces an increase in the expression of the gene coding for the serotonin transporter, associated with a subsequent elevation in the uptake of serotonin. This stimulatory effect, produced upon incubation with cortisol in vitro, was observed in peripheral blood lymphocytes from normal subjects. In the present work we investigated the cortisol-induced increase in serotonin uptake in lymphocytes from hypercortisolemic patients, including subjects with major depressive disorder $(n=8)$, and subjects with generalized anxiety disorder $(n=12)$, in comparison with a control group of normal healthy subjects $(n=8)$. A significant increase in serotonin uptake $(+37 \%+14, M+S D)$ was observed in the control group, whereas neither the generalized anxiety disorder nor the major depression group exhibited changes in serotonin uptake upon incubation with cortisol. It is likely that under chronic stress or depression, the capacity for increase in serotonin transporter has reached its limit due to the chronically elevatedblood cortisol level. The physiological and diagnostic implications of this observation are discussed.
\end{abstract}

The role of chronic psychosocial stress in the origin and development of depression has been thoroughly investigated, producing a large body of evidence in support of this association (Breier et al., 1987; Dolan, Calloway, Fonagy, De Souza, \& Wakeling, 1985; Gold, Goodwin, \& Chrousos, 1988a; Post, 1992). The response of the central nervous system (CNS) to psychosocial stressors is predominantly through activation of the locus coeruleus/sympathetic nervous system, with the consequent release of noradrenaline and adrenaline, and the limbic/hypothalamo-pituitaryadrenal (HPA) system, with the consecutive release of corticotropin-releasing hormone ( $\mathrm{CRH})$, adrenocorticotropin hormone (ACTH), and glucocorticoids, particularly cortisol (Akil \& Morano, 1995; Chrousos \& Gold, 1992).

Correspondence should be addressed to G. E. Tafet, Instituto de Investigaciones Bioquímicas, Fundación Campomar, Lab. 109, Universidad de Buenos Aires, Av. Patricias Argentinas 435, Buenos Aires 1425, Argentina.
The characteristics of this adaptive response in each individual case will be determined by the attributes of the stressor itself, its appraisal, and the resulting coping strategies (Lazarus, 1966; Lazarus \& Folkman, 1984). Therefore, chronic stress due to intensive or prolonged exposure to stressors, combined with the inadequacy to cope or the perceived loss of controllability, will be characterized by the persistent activation of the HPA system (Croes, Merz, \& Netter, 1993; Henry, 1992). The normal activity of the HPA axis, characterized by wide circadian variations, with morning zeniths and evening nadirs, is regulated by diurnal excitatory inputs, stress-induced stimulation, and various negative-feedback loops, mediated by $\mathrm{CRH}, \mathrm{ACTH}$, and to a large extent by cortisol (Dallman et al., 1994; KellerWood \& Dallmam, 1984; Munck, Guyre, \& Holbrook, 1984). However, the ability of cortisol to regulate its own production may be impaired during chronic stress (Halbreich, Asnis, Schindledecker,Zumoff, \& Swami Nathan, 1985), resulting in sustained increase in plasma cortisol level (Ottenweller, Natelson, Pitman, \& Drastal, 1989). 
Hence, the persistent activation of the HPA axis will lead to dysregulation, characterized by a flattened circadian curve, mostly due to elevations in the evening nadir and discrete decline in the morning zenith (Chrousos \& Gold, 1998). These characteristics of chronic stress have been also observed in major depression, representing one of the most consistent findings in biological psychiatry (Gold, Goodwin, \& Chrousos, 1988b).

The origin and development of depression is also associated with dysfunction of the serotonergic system in the CNS (Henninger, 1995; Maes \& Meltzer, 1995; Meltzer \& Lowy, 1987). At the molecular level, this system is regulated by the serotonin transporter, which by reuptaking the released neurotransmitter, is responsible for keeping the effective concentrations of serotonin at the synaptic cleft (Amara \& Kuhar, 1993; Barker \& Blakely, 1995). Therefore, the serotonin transporter became an important element in psychopharmacology as the target of most antidepressants (Hoffman, Mezey, \& Brownstein, 1991).

We have recently shown that cortisol enhances the uptake of serotonin in vitro by human peripheral blood lymphocytes (PBLs; Tafet, Toister-Achituv, \& Shinitzky, 2001) due to induction of synthesis of the serotonin transporter. Since the serotonin transporter of human PBLs is identical to that of the neuronal tissues (Faraj, Olkowsky, \& Jackson, 1994, 1997), the hyperactivity of serotonin uptake induced by cortisol could provide a link between stress and depression (Tafet et al., 2001). In addition, we also reported that PBLs from hypercortisolemic rabbits presented an effect similar to that observed with human PBLs upon incubation in vitro with cortisol.

Here we present a study on the effect of cortisol on the uptake of serotonin by PBLs from depressed and a subpopulation of chronically stressed patients. We hypothesized that hypercortisolism would be implicated in the impairment of the serotonergic neurotransmission, due to increased serotonin reuptake, and this mechanism could provide a molecular link between the hyperfunctionalHPA axis of the chronically stressed, and the dysfunctional serotonergic system of the depressive.

\section{METHOD}

A total of 28 subjects (12 males and 16 females), age 36 to 60 years $(M=44.8$ years, $S D=7.2)$, participated in the study. Depressive subjects were recruited among outpatients of the Department of Psychiatry, Hospital Zubizarreta, Buenos Aires. Subjects with generalized anxiety disorder were recruited among outpatients and hospital staff members. Normal healthy volunteers, recruited among staff members of the hospital, were examined and consequently considered as a control group. Diagnostic classification was based on clinical criteria corresponding to the fourth edition of the Diagnostic and Statistical Manual of Mental Disorders (DSM-IV) (American Psyciatric Association [APA], 1994). The protocol was evaluated and approved by the ethical committee of the hospital, and all the subjects gave voluntary informed consent for participation in the study.

All subjects were interviewed and accordingly diagnosed by consensus between two psychiatrists (G.E.T. and S.S.R.). Initially, in order to encourage cooperation among clinicians and patients in a general hospital, the assessment and diagnosis were performed using the Hospital Anxiety and Depression Scale (HAD-S; Zigmond \& Snaith, 1983). But after it failed to provide all the data necessary to conclude accurate diagnostic criteria, this simpler scale was replaced by a more comprehensive state-of-the-art approach. Therefore, all potential patients were then evaluated according to the Structured Clinical Interview for DSM-IV Axis I disorders (SCID; First, Spitzer, Gibbon, \& Williams, 1997) to establish the diagnostic categories and determine exclusionary diagnoses. Exclusion criteria were any lifetime history of mania or psychosis, or a substance abuse history within the past 6 months.

Patients diagnosed as depressive met the $D S M-I V$ criteria for major depressive disorder (MDD), and the severity of depressive symptoms was assessed according to the Hamilton Depression Rating Scale (HAM-D; Hamilton, 1960). In order to restrict the sample to welldefined criteria representative of a specific chronic stress syndrome, only patients suffering from generalized anxiety disorder (GAD) were selected. Therefore, these patients met the $D S M-I V$ criteria for GAD, and their severity of anxiety-related symptoms was assessed according to the Hamilton Anxiety Rating Scale (HAM-A; Hamilton, 1959). Out of this sample, 8 subjects ( 3 male and 5 female, mean age $=48.9$ years, $S D=9.2$ ) met the diagnostic criteria for MDD (HAM-D score $=25.3, S D=3.9$, HAM-A score $<14$ ), and 12 subjects ( 5 male and 7 female, mean age $=43.4$ years, $S D=5.9$ ) met diagnostic criteria for GAD (HAM-A score $=19.5, S D=5.6$, HAM-D score $<14$ ). Control subjects ( 4 male and 4 female, mean age $=$ 42.7 years, $S D=5.9$ ) were considered physically healthy and did not present any psychiatric condition. Their HAM-D and HAM-A scores did not reach signif icant values (both scales scored less than 14 points). None of the subjects in the three groups were under treatment with antidepressants, neuroleptics, or glucocorticoids, nor had they had any substance abuse within the past 6 months.

Two peripheral blood samples were drawn from each subject at 8:00 and 16:00 hours of the same day, in the clinical laboratory of the Zubizarreta Hospital. Plasma cortisol concentrations were determined by chemiluminiscence with the use of a standard, commercially available competitive immunoassay (Diagnostic Products Corp.). The normal range was taken as $7-25 \mathrm{ng} / \mathrm{dl}$ for the morning and 2-9 ng/dl for the afternoon (Sapse, 1997).

For lymphocyte separation, both blood samples from each patient were centrifuged at $900 \mathrm{rpm}$ for $20 \mathrm{~min}$, to separate the platelet rich plasma, which was discarded. The remaining blood fraction was layered over $8 \mathrm{ml}$ of Ficoll-Paque (Pharmacia) and centrifuged at $1,800 \mathrm{rpm}$ for $20 \mathrm{~min}$. The mononuclear leucocyte layer was further processed as described (Faraj et al., 1994) for purification of lymphocytes. The final lymphocyte sample was resuspended in RPMI-1640 medium containing 10\% FCS, $2 \mathrm{mM} \mathrm{L-glutamine,} 1 \mathrm{mM}$ sodium pyruvate, nonessential aminoacids, antibiotics, and the number of lymphocytes was adjusted to $10^{7}$ cells $/ \mathrm{ml}$. The lymphocytes from each subject were distributed in micro titer plates (Nunc), $1 \mathrm{ml}$ per sample, to be incubated in the presence or absence of $10^{-8} \mathrm{M}$ cortisol (as described in Tafet et al., 2001), and kept in $5 \% \mathrm{CO}_{2}$ humidified incubator at $37^{\circ} \mathrm{C}$ for $48 \mathrm{~h}$.

Uptake of serotonin (5-HT) was assayed with tritiated 5-HT $\left(\left[{ }^{3} \mathrm{H}\right]-\right.$ 5-HT, NEN) essentially as reported in Faraj et al. (1991, 1994). Cortisol-treated and control samples were incubated for $15 \mathrm{~min}$ at $37^{\circ} \mathrm{C}$ in the presence of $10^{-6} \mathrm{M}$ 5-HT (Sigma), doped with $\left[{ }^{3} \mathrm{H}\right]-5-\mathrm{HT}$ in 1.5-ml microtubes (Eppendorf ). The uptake was terminated by centrifugation at $14,000 \mathrm{rpm}$ for $5 \mathrm{~min}$ in a microfuge at $4^{\circ} \mathrm{C}$. The pellet was washed with $1 \mathrm{ml}$ of cold PBS, centrifuged, suspended in $200 \mu \mathrm{l}$ of $1-\mathrm{M} \mathrm{NaOH}$, incubated overnight at $37^{\circ} \mathrm{C}$, and neutralized with $20 \mu \mathrm{l}$ of concentrated $\mathrm{HCl}$. The obtained homogenates were transferred to scintillation vials containing $2 \mathrm{ml}$ of scintillation fluid and scored for radioactivity.

Differences in serotonin uptake between the three groups were tested by a one-way analysis of variance (ANOVA) with clinical classification as the independent variable, followed by a post hoc Scheffé test. Differences in cortisol levels between the three groups were also 
tested by a one-way ANOVA with clinical classification as an independent variable; correlations between cortisol levels and serotonin uptake were also tested by a one-way ANOVA with cortisol level as the independent variable, followed by a post hoc Scheffé test.

\section{RESULTS}

Plasmatic cortisol concentrations were determined in the morning (8:00 a.m.) and afternoon (4:00 p.m.) as described in the Method section. For the control group we found a mean cortisol concentration of $18.8 \mathrm{ng} / \mathrm{dl}(S D=$ $3.3)$ in the morning, and $8.6 \mathrm{ng} / \mathrm{dl}(S D=0.9)$ in the afternoon. The ratio between these values $(4: 00$ p.m./ 8:00 a.m. plasma cortisol) was less than 50\% ( $M=44.6 \%$, $S D=5.7$ ). These values fall in the normal range (Sapse, 1997). For the GAD group, a significant increase of cortisol concentrations in the afternoon was observed $(M=$ $17.7, S D=4.4)$, whereas in the morning it was at the upper normal level $(M=25.2, S D=5.2)$. The ratio between these values was found to be higher than $50 \%(M=68.9 \%$, $S D=11.9$ ), suggesting a blunted circadian rhythm of cortisol secretion. In patients with MDD, we observed a significant increase of cortisol concentrations in the afternoon $(M=14.8, S D=2.8)$, but not in the morning $(M=$ $19.7, S D=2.1)$. The ratio between these values was significantly higher than $50 \%(M=74.8 \%, S D=10.3)$, also suggesting a flattened curve of cortisol secretion. Statistical analysis of the correlation between cortisol levels with clinical classification as an independent variable revealed a significant difference for the afternoon values $[F(2)=$ $16.35, p<.0001]$, but a lower significance for the morning values $[F(2)=7.13, p<.05]$. The Scheffé test indicated a significant difference for the afternoon levels of cortisol between the GAD and the control group $(p<$ $.0001)$, and the MDD and the control group $(p<.0001)$. Differences between the results corresponding to the GAD and MDD groups were not statistically significant. For the morning levels of cortisol, the differences between the groups were not significant either.

The effect of cortisol on the serotonin uptake by PBLs was determined in vitro, as shown in Figure 1. PBLs from control subjects responded in vitro to the incubation with cortisol by a significant increase in the uptake of serotonin $(M=137.3 \%, S D=14.2)$, which falls in a range similar to that reported under similar conditions (Tafet et al., 2001). In contrast to that, the in vitro effect of cortisol on the uptake of serotonin was not observed in PBLs from GAD subjects $(M=101.1 \%, S D=10.7)$ or MDD subjects $(M=$ $101.5 \%, S D=10.5)$. The Scheffé test indicated a significant difference between the GAD and the control group $(p<.0001)$, and the MDD and the control group $(p<$ $.0001)$. Differences between the results corresponding to the GAD and MDD groups were not statistically significant. The correlation between cortisol levels and serotonin uptake was also tested by a one-way ANOVA with afternoon level of cortisol as an independent variable, revealing a significant correlation between the differences observed in vitro in the uptake of serotonin, and the plasma levels of cortisol in the afternoon of the different groups $[F(1)=52.49, p<.0001]$.

The increase in the afternoon level of cortisol was significantly correlated with a reduced response in the uptake of serotonin upon incubation with cortisol in vitro $\left[r^{2}=\right.$ $.43, F(1)=19.8, p<.001]$, as shown in Figure 2.

\section{DISCUSSION}

It has been shown that chronic stress plays a critical role in the origin and development of depression (Breier et al., 1987; Dolan et al., 1985; Gold et al., 1988a; Post, 1992). This association has been extensively investigated in various studies, most of them focusing on the activation of the HPA axis (Dinan, 1994; Peeters \& Broekkamp, 1994), which constitutes the basic neuroendocrine reaction in the adaptive response to stress (Axelrod \& Reisine, 1984). Chronic activation of the stress system, due to intensive or prolonged exposure to stressors, combined with the inadequacy to cope, or the perceived loss of controllability, will be therefore characterized by the persistent activation of the HPA system and elevation of circulating cortisol (Croes et al., 1993; Henry, 1992).

Various regions in the CNS are important targets for glucocorticoids, particularly the hippocampus (McEwen, Davis, \& Parsons, 1979). Hence, neuronal damage in the hippocampus, produced by excessive and prolonged exposure to cortisol, and the consecutive impairment in the control feedback at the suprahypothalamiclevels, may account for the inability of cortisol to regulate its own production during chronic stress (Chrousos \& Gold, 1998; McEwen, 1998). In addition, these limbic structures are in turn modulated by multiple neural pathways, including serotonergic input from the raphe nuclei, particularly the median raphe nucleus, which innervates various forebrain structures, most prominently the hippocampus (Azmitia \& Whitaker-

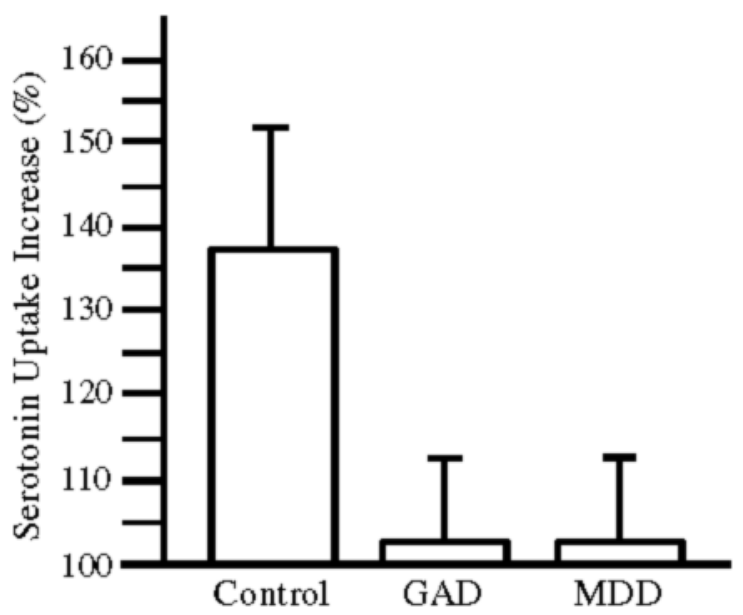

Figure 1. Percent increase of $\left[{ }^{3} \mathrm{H}\right]-5 \mathrm{HT}$ uptake by PBLs of patients with generalized anxiety disorder (GAD), major depressive disorder (MDD), and control subjects, after incubation for $48 \mathrm{~h}$ with $10^{-8} \mathrm{M}$ cortisol. 


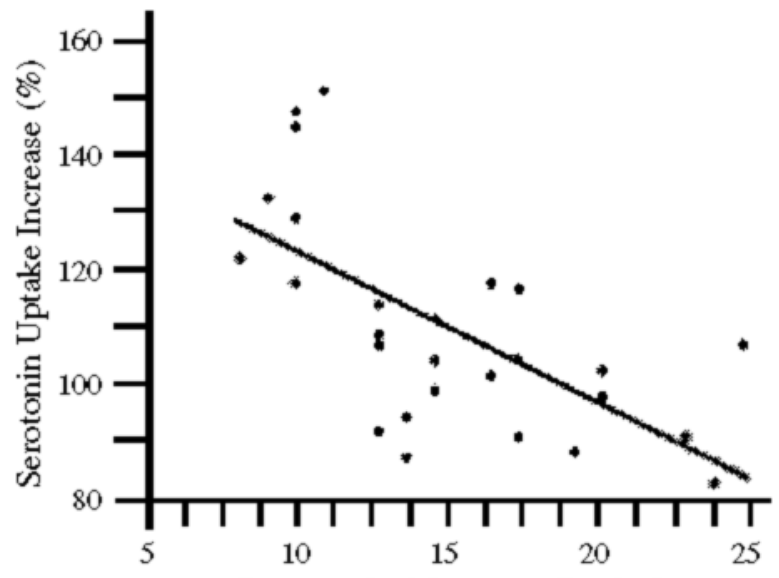

Increase in Afternoon Cortisol

Figure 2. Significant relationship between the increase in the afternoon levels of cortisol and the reduced $\left[{ }^{3} \mathrm{H}\right]-5 \mathrm{HT}$ uptake upon in vitro incubation with cortisol $\left[r^{2}=.43, F(1)=19.8, p<\right.$ $.001]$.

Azmitia, 1995; Deakin, 1991). This system was implicated in the tolerance to adverse stimuli and negative emotional experiences (Smelik, 1987). Moreover, dysfunction of this serotonergic pathway was also associated with stressinduced learned helplessness and subsequent depression (Deakin, 1991).

At the molecular level, the serotonergic neurotransmission is regulated by the serotonin transporter, which is responsible for the reuptake of the released neurotransmitter, therefore determining the effective concentrations of serotonin in the synaptic cleft (Amara \& Kuhar, 1993; Barker \& Blakely, 1995). Hence, the serotonin transporter is the target of most antidepressants, including tricyclics and selective-serotonin-reuptake-inhibitors (Hoffman et al., 1991; Kanner \& Schuldiner, 1987), which by restraining the reuptake process maintain increased concentrations of the neurotransmitter at the synaptic cleft.

In our previous study (Tafet et al., 2001), we demonstrated that in vitro incubation of PBLs from normal human subjects with cortisol induces a significant increase of serotonin uptake, which was attributed to an increased expression of the serotonin transporter gene. This effect was associated with the GR-cortisol complex, which regulates transcriptional responses upon binding to a hormone response element, located in the promoter region of the target genes (Reichel \& Jacob, 1993). The gene encoding the human serotonin transporter was shown to be identical in neurons and lymphocytes (Faraj et al., 1994, 1997), and various studies indicate that the analogy in the uptake of neurotransmitters between both types of cells is actually valid (Faraj et al., 1991, 1994, 1997; Grodzicki et al., 1990; Halbach \& Henning, 1989). In addition, we also reported that PBLs from rabbits previously treated in vivo with cortisol presented an effect similar to that observed in human PBLs upon incubation with cortisol in vitro. Moreover, before injection of cortisol, the PBLs of each individual rabbit responded to the presence of cortisol in vitro by an increase in serotonin uptake of $20 \%-$ $80 \%$, a range similar to that observed with human PBLs. When PBLs of hypercortisolemic rabbits were tested, no further increase in serotonin uptake upon incubation with cortisol in vitro was observed. These findings support the possibility that the increase in serotonin transporter upon prolonged presence of abnormally high levels of cortisol in vivo reaches a saturation level that could not be further increased upon incubation in vitro with cortisol (Tafet et al., 2001).

In the present study we observed a substantial increase in the afternoon cortisol level in the blood of patients with GAD and MDD. This elevation has been described previously as a characteristic expression of the hypercortisolism observed in the chronically stressed (Chrousos \& Gold, 1998) and the endogenously depressed (Halbreich et al., 1985). As with our results in hypercortisolemic rabbits, incubation with cortisol in vitro of PBLs of these hypercortisolemic subjects did not show any increase in the uptake of serotonin. On the other hand, PBLs from control subjects exhibited a substantial increase in the uptake of serotonin upon $48 \mathrm{~h}$ incubation with cortisol in vitro, as we previously observed (Tafet et al., 2001).

With respect to GAD, it should be noted that the DSM$I V$ registers different categories of anxiety disorders, including a specific acute stress disorder, and different syndromes characterized by a chronic course. GAD is characterized by excessive anxiety and worry about various life events, implying an important source of subjective distress, during a period of at least 6 months (APA, 1994). Therefore, even if there is no specific category for chronic stress in the $D S M-I V$, we can consider GAD essentially as a chronic stress disorder. Hence, since not every patient with chronic stress is in the GAD category, but all patients with GAD are chronically stressed, we can consider these patients as a subgroup of the chronically stressed, which in addition fulfill specific criteria for GAD.

Considering the facts that the serotonin transporters in PBLs and neuronal tissues are identical (Faraj et al., 1994, 1997) and that the increase in cortisol level is actually systemic, the results of the present study strongly suggest that dysregulation of the HPA axis, with the consequent hypercortisolism, is involved in the observed differential rate of serotonin uptake. The proposed interaction between the HPA axis and the serotonergic function is in line with the hypothesis that attributes hypercortisolism to mood changes observed in depression (Dinan, 1994; Peeters \& Broekkamp, 1994). In addition, the observation that PBLs from both groups of hypercortisolemic patients presented a similar pattern of response (Figure 1) supports the notion that depression could be interpreted not only as a consequence but also as a chronic stress disorder by itself. Whether certain stressful life events will precipitate depression in some individuals and not in others will depend on psychobiological background (Dinan, 1994; Dolan et al., 1985; Post, 1992) and psychosocial variables, such as attributional style (Abramson, Seligman, 
$\&$ Teasdale, 1978) and a set of cognitive patterns that direct the patient to assume negative views of himself/herself, his/her experiences, and his/her future (Beck, Rush, Shaw, $\&$ Emery, 1979). These patterns are organized in a stable and global manner and are validated by subsequent cognitive distortions that maintain the belief in these negative features.

The link between hypercortisolemia and impaired serotonergic activity is in line with both psychobiological and cognitive models of depression, and further supports the notion that specific interventions, directed at normalization of the HPA system, could represent a potential strategy to prevent the development of depression in chronically stressed subjects. This could be achieved through pharmacologic interventions, either directly by means of anticortisol drugs (Reus, Wolkowitz, \& Frederick, 1997; Sapse, 1997; Thakore \& Dinan, 1995; Wolkowitz et al., 1993) or indirectly through psychotherapeutic approaches.

Our findings also bear some biochemical diagnostic potential. Direct measurement of $\left[{ }^{3} \mathrm{H}\right]-5 \mathrm{HT}$ uptake by PBLs requires a series of standardization steps that are impractical, whereas a comparative test where $\left[{ }^{3} \mathrm{H}\right]-5 \mathrm{HT}$ uptake is measured in PBLs after incubation with and without cortisol is straightforward. In such a test the percent increase in $\left[{ }^{3} \mathrm{H}\right]-5 \mathrm{HT}$ could serve as an absolute parameter for deviation from normality. In future work, we plan to verify our findings in larger groups and to include different groups of chronically stressed subjects. In addition, the effects of abnormal levels of physiological factors other than cortisol on serotonin uptake will be assessed.

\section{REFERENCES}

Abramson, L. Y., Seligman, M., \& Teasdale, L. D. (1978). Learned helplessness in humans: Critique and reformulation. Journal of Abnormal Psychology, 87, 49-78.

AKIL, H. A., \& Morano, M. I. (1995). Stress. In F. E. Bloom \& D. J. Kupfer (Eds.), Psychopharmacology: The fourth generation of progress (pp. 773-785). New York: Raven.

Amara, S. G., \& Kuhar, M. J. (1993). Neurotransmitter transporters: Recent progress. Annual Review of Neuroscience, 16, 73-93.

American Psychiatric Association (1994). Diagnostic and statistical manual of mental disorders (4th ed.). Washington, DC: Author.

Axelrod, J., \& Reisine, T. D. (1984). Stress hormones: Their interaction and regulation. Science, 224, 452-459.

Azmitia, E. C., \& Whitaker-Azmitia, P. M. (1995). Anatomy, cell biology, and plasticity of the serotonergic system: Neuropsychopharmacological implications for the actions of psychotropic drugs. In F. E. Bloom \& D. J. Kupfer (Eds.), Psychopharmacology: The fourth generation of progress (pp. 443-449). New York: Raven.

BARKER, E. L., \& BLAKELY, R. D. (1995). Norepinephrine and serotonin transporters: Molecular targets of antidepressant drugs. In F. E. Bloom \& D. J. Kupfer (Eds.), Psychopharmacology: The fourth generation of progress (pp. 321-334). New York: Raven.

Beck, A. T., Rush, A. J., Shaw, B. F., \& Emery, G. (1979). Cognitive therapy of depression. New York: Guilford.

Breier, A., Albus, M., Pickar, D., Zahn, T. P., Wolkowitz, O. M., \& PAUl, S. M. (1987). Controllable and uncontrollable stress in humans: Alterations in mood and neuroendocrine and psychophysiologicalfunction. American Journal of Psychiatry, 144, 1419-1425.

Chrousos, G. P., \& Gold, P. W. (1992). The concepts of stress and stress system disorders. Journal of the American Medical Association, 267, 1244-1252.
Chrousos, G. P., \& Gold, P. W. (1998). A healthy body in a healthy mind-and vice versa - the damaging power of "uncontrollable" stress. Journal of Clinical Endocrinology \& Metabolism, 83, 18421845.

Croes, S., Merz,P., \& Netter, P. (1993). Cortisol reaction in success and failure condition in endogenous depressed patients and controls. Psychoneuroendocrinology, 18, 23-35.

Dallman, M. F., Akana, S. F., Levin, N., Walker, C.-D., Bradbury, M. J., Suemaru, S., \& Scribner, K. S. (1994). Corticosteroids and the control of function in the hypothalamo-pituitary-adrenal(HPA) axis. In E. R. de Kloet, E. C. Azmitia, \& P. W. Landfield (Eds.), Brain corticosteroid receptors: Studies on the mechanism, function, and neurotoxicity of corticosteroid action (Annals of the New York Academy of Sciences, Vol. 746, pp. 22-33). New York: New York Academy of Sciences.

DeAKIN, J. F. W. (1991). Distinct roles of 5HT subsystems in panic, anxiety and depression. In G. Racagni, N. Brunello, \& T. Fukuda (Eds.), Biological psychiatry (Vol. 1, pp. 305-307). Amsterdam: Elsevier.

Dinan, T. G. (1994). Glucocorticoids and the genesis of depressive illness: A psychobiological model. British Journal of Psychiatry, 164, 365-371.

Dolan, R. J., Calloway, S. P., Fonagy, P., De Souza, F. V. A., \& WAKELING, A. (1985). Life events, depression and hypothalamicpituitary-adrenal axis function. British Journal of Psychiatry, 147, 429-433.

FaraJ, B. A., Olkowsky, Z. L., \& Jackson, R. T. (1991). Binding of [3H]-dopamine to human lymphocytes: Possible relationship to neurotransmitter uptake sites. Pharmacology, 42, 135-141.

FARAJ, B. A., Olkowsky, Z L., \& Jackson, R. T. (1994). Expression of a high-affinity serotonin transporter in human lymphocytes. International Journal of Immunopharmacology, 16, 561-567.

FARAJ, B. A., OlKowsky, J. L., \& Jackson, R. T. (1997). Prevalence of high serotonin uptake in lymphocytes of abstinent alcoholics. Biochemical Pharmacology, 53, 53-57.

First, M. B., Spitzer, R. L., Gibbon, M., \& Williams, J. B. W. (1997). Structured clinical interview for DSM-IV: Axis I Disorders. Clinician Version. Washington, DC: American Psychiatric Press.

Gold, P. W., Goodwin, F. K., \& Chrousos, G. P. (1988a). Clinical and biochemical manifestations of depression: Relation to neurobiology of stress (Part I). New England Journal of Medicine, 319, 348-353.

Gold, P. W., Goodwin, F. K., \& Chrousos, G. P. (1988b). Clinical and biochemical manifestations of depression: Relation to neurobiology of stress (Part II). New England Journal of Medicine, 319, 413-420.

Grodzicki, J., Pardo, M., Schved, G., Schlosberg, A., Fuchs, S., \& Kanety, H. (1990). Differences in $3 \mathrm{H}$-spiperone binding to peripheral blood lymphocytes from neuroleptic responsive and nonresponsive schizophrenic patients. Biological Psychiatry, 27, 1327 1330.

Halbach, M., \& Henning, U. (1989). Abnormal glucocorticoid dependent increase of spiperone binding sites on lymphocytes from schizophrenics in vitro. Pharmacopsychiatry, 22, 168-173.

Halbreich, U., Asnis, G. M., Schindledecker, R., Zumoff, B., \& SWAMi NATHAN, R. (1985). Cortisol secretion in endogenous depression: I. Basal plasma levels. Archives of General Psychiatry, 42, 904-908.

Hamilton, M. (1959). The assessment of anxiety states by rating. British Journal of Medical Psychology, 32, 50.

Hamilton, M. (1960). A rating scale for depression. Journal of Neurology, Neurosurgery, \& Psychiatry, 23, 56-62.

HenNinger, G. R. (1995). The role of serotonin in clinical disorders. In F. E. Bloom \& D. J. Kupfer (Eds.), Psychopharmacology: The fourth generation of progress (pp. 471-482). New York: Raven.

HENRY, J. P. (1992). Biological basis of the stress response. Integrative Physiology \& Behavioral Science, 27, 66-83.

Hoffman, B. J., Mezey, E., \& Brownstein, M. J. (1991). Cloning of a serotonin transporter affected by antidepressants. Science, 254, 579580 .

KAnNer, B. I., \& Schuldiner, S. (1987). Mechanism of transport and storage of neurotransmitters. CRC Critical Reviews in Biochemistry, 22, 1-38.

Keller-Wood, M. E., \& Dallmam, M. F. (1984). Corticosteroid inhibition of ACTH secretion. Endocrine Reviews, 5, 1-24. 
Lazarus, R. S. (1996). Psychological stress and the coping process. New York: McGraw-Hill.

LAZARUs, R. S., \& Folkman, S. (1984). Stress, appraisal and coping. New York: Springer-Verlag.

MaEs, M., \& Meltzer, H. Y. (1995). The serotonin hypothesis of major depression. In F. E. Bloom \& D. J. Kupfer (Eds.), Psychopharmacology: The fourth generation of progress (pp. 933-943). New York: Raven.

McEwen, B. S. (1998). Protective and damaging effects of stress mediators. New England Journal of Medicine, 338, 171-179.

McEwen, B. S., Davis, P. G., \& Parsons, B. (1979). The brain as a target for steroid hormone action. Annual Review of Neuroscience, 2 , 65-112.

Meltzer, H. Y., \& Lowy, M. T. (1987). The serotonin hypothesis of depression. In H. Meltzer (Ed.), Psychopharmacology: The third generation of progress (pp. 513-526). New York: Raven.

Munck, A., GuYre, P. M., \& Holbrook, N. J. (1984). Physiological functions of glucocorticoids in stress and their relation to pharmacological actions. Endocrine Reviews, 5, 25-55.

Ottenweller, J. E., Natelson, B. H., Pitman, D. L., \& Drastal, S. D. (1989). Adrenocortical and behavioral responses to repeated stressors: Toward an animal model of chronic stress and stressrelated mental illness. Biological Psychiatry, 26, 829-841.

Peeters, B. W. M. M., \& BroekKamp, C. L. E. (1994). Involvement of corticosteroids in the processing of stressful life-events: A possible implication for the development of depression. Journal of Steroid Biochemistry \& Molecular Biology, 49, 417-427.

Post, J. M. (1992). Transduction of psychosocial stress into the neuro- biology of recurrent affective disorders. American Journal of Psychiatry, 149, 999-1010.

ReICHeL, R. R., \& JACOB, S. T. (1993). Control of gene expression by lipophilic hormones. Federation of American Societies for Experimental Biology Journal, 7, 427-436.

Reus, V. I., Wolkowitz, O. W., \& Frederick, S. (1997). Antiglucocorticoid treatments in psychiatry. Psychoneuroendocrinology, 22 (Suppl. 1), S121-S124.

SAPSE, A. (1997). Cortisol, high cortisol diseases and anticortisol therapy. Psychoneuroendocrinology, 22 (Suppl. 1), S3-S10.

Smelik, P. G. (1987). Adaptation and brain function. Progress in Brain Research, 72, 3-9.

Tafet, G. E., Toister-Achituv, M., \& Shinitzky, M. (2001). Enhancement of serotonin uptake by cortisol: A possible link between stress and depression. Cognitive, Affective, \& Behavioral Neuroscience, 1, 96-104.

Thakore, J. H., \& Dinan, T. (1995). Cortisol synthesis inhibition: A new treatment strategy for the climical and endocrine manifestations of depression. Biological Psychiatry, 37, 364-368.

Wolkowitz, O. M., Reus, V. I., Manfredi, F., Ingbar, J., BrizenDINE, L., \& WEINGARTNER, H. (1993). Ketoconazole administration in hypercortisolemic depression. American Journal of Psychiatry, 150, 810-812.

Zigmond, A. S., \& SNaith, R. P. (1983). The hospital anxiety and depression scale. Acta Psychiatrica Scandinavica, 67, 361-370.

(Manuscript received December 10, 2000; revision accepted for publication October 29, 2001.) 